\title{
A New Method for Determining Horizontal Impact Load Based on Rotational Speed of Aircraft Wheel in Landing Gear Drop Test
}

\author{
Wang Huaitao ${ }^{1}$, Wu Dafang ${ }^{2, a}$ and Wang Feng ${ }^{3}$ \\ ${ }^{1,2,3}$ School of Aeronautic Science and Engineering, Beihang University, No. 37 Xueyuan Road, Haidian District, \\ Beijing, China.
}

\begin{abstract}
The maximum horizontal impact load experienced by the aircraft landing gear wheel during landing is an important parameter in landing gear safety design and performance analysis. Using a non-contact photoelectric testing method, this paper experimentally obtained the dynamic data of the instantaneous rotational speed for an aircraft wheel when it contacts the platform. Moreover, the kinetic relationship between the transient rotational speed of the aircraft wheel and the force of horizontal impact was established. According to the change data measured from the transient rotational speed of the aircraft wheel, the maximum horizontal impact load at the instant of aircraft contact with the platform was calculated. To verify the accuracy of this method, in which changes in rotational speed determine the horizontal impact load, verification equipment without lateral constrains was established to test the horizontal impact load of the aircraft wheel. The experimental results showed that the horizontal impact load determined by the transient rotational speed of aircraft wheel is consistent with the results obtained by self-built measurement equipment without lateral constrains.
\end{abstract}

\section{Introduction}

The landing gear is an extremely important component of an aircraft structure. In the moment of the aircraft landing, the landing gear hits the ground with a high speed and the wheels immediately transform from a static state to a high-speed rotation state. During this interval, the change of impact load in the horizontal direction experienced by the aircraft wheel is rapid and complex. In addition, a direct relationship exists between changes in the horizontal impact load on the aircraft wheel and the design of key components, such as the aircraft wheel brakes, bumpers, and shimmy dampers for the rockers, all of which also affect performance studies, such as the strength and stiffness of the landing gear, brake damping efficiency, power absorption, and aircraft wheel spin-up and springback[1].Therefore, determining the horizontal impact load of the landing gear wheel upon contact with the ground through landing gear drop test, plays a very important role in the strength analysis and safety design of landing gear systems.

At present, the wheel horizontal impact load is generally measured by the force sensor. In aircraft landing gear drop tests, a traditional method in which force sensors are horizontally mounted on the end of a drop test platform is employed[2-4]. The aircraft wheel horizontal impact load upon contact

\footnotetext{
${ }^{a}$ Corresponding author : wdf1950@163.com
} 
with platform is recorded by the horizon tally mounted force sensor at the side of drop test platform. However, the spherical connection mode of the vertical support column of the landing platform causes the restriction of the free movement of the landing platform in the horizontal direction, which has an influence on the accuracy of horizontal load measurement. The inertia force of the platform is considered by Shi Haiwen et al. and the spherical connection mode between the drop test platform and the vertical sensor is replaced with a sliding connection mode, which can improve the accuracy of the measured result to some extent[5]. The serious lateral shake will occur when the aircraft wheel touches the platform, so lateral friction between the slide rails restricts the free movement of the drop test platform in the horizontal plane. Thus, the accuracy of the horizontal impact force of aircraft wheel upon contact with the platform is affected. As a result, researchers have been searching for a better method for measuring the landing gear horizontal impact force.

This paper proposes a new non-contact measuring method with practical application that determines the landing gear horizontal impact load in drop tests by measuring and recording the change of aircraft wheel transient rotational speed. First, the dynamic relationship between the transient speed change and the horizontal impact force is established, and then the maximum horizontal impact load of the aircraft wheel is calculated based on the measured data of the aircraft wheel transient rotational speed. The proposed method can avoid the interactive effects caused by the design of landing gear drop platform, which is used to measure the different direction impact loads.

\section{Determining the horizontal impact load}

First, the kinetic relationship between the horizontal impact load and the transient rotational speed of the aircraft wheel must be established. Next, the change in the transient rotational speed of the aircraft wheel at the moment of impact with the ground is obtained through the drop test apparatus and the aircraft wheel transient rotational speed measurement system. Then, the size of the horizontal impact load at the instant of aircraft wheel contact with the ground is established according to the kinetic relationship between the transient rotational speed of the aircraft wheel and the force of horizontal impact.

\subsection{Kinetic relationship between the horizontal impact load and the transient rotational speed of the aircraft wheel}

The horizontal force experienced by the aircraft wheel during high-speed rotation in the drop tests, when dropped into contact with the platform, is shown in Figure 1.

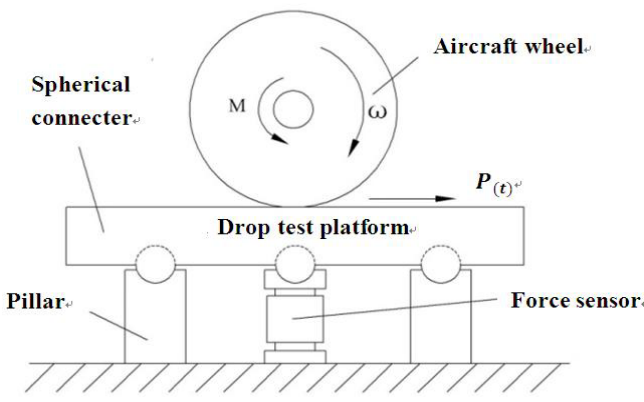

Figure 1 Mechanical model of an aircraft wheel in contact with the platform

The Figure 1 shows the horizontal impact load is related to the friction between the aircraft wheel and platform, as well as the friction between the aircraft wheel and axle. Given $\omega$ as the rotational speed of the aircraft wheel, $\frac{2 \pi}{60} \omega$ is the angular velocity of the aircraft wheel. According to the theorem of the moment of momentum, the acquirable kinetic relationship between the horizontal impact load and the transient rotational speed of the aircraft wheel is 


$$
\frac{d\left(\frac{2 \pi}{60} \omega I\right)}{d t}=P(t) R(t)+M
$$

In the formula, $P(t)$ is the horizontal impact load of the aircraft wheel, $\mathrm{t}$ is time, $R$ is the radius of the aircraft wheel, $M$ is the friction torque of the aircraft axle, and $I$ is the moment of inertia of the aircraft axle. Under the condition of this experiment, the above formula can be converted into the following form

$$
\frac{2 \pi}{60} \frac{d \omega}{d t} I=P(t) R+M
$$

In the formula, $\frac{2 \pi}{60} \frac{d \omega}{d t}$ is the angular acceleration while aircraft wheel rotating.

The calculation formula for the horizontal impact load of the aircraft wheel, $P(t)$, is

$$
P(t)=\left(\frac{2 \pi}{60} \frac{d \omega}{d t}-\frac{2 \pi}{60} \frac{d \omega_{0}}{d t}\right) I / R
$$

Analysis of Formula (3) shows the amount of change over time of the measured transient rotational speed, $\omega$, in the drop test when the aircraft wheel touches the platform and the amount of attenuation change over time in the aircraft wheel rotational speed, $\omega_{0}$, in a state of free rotation. Substitution into Formula (3) can be done to immediately calculate the changes over time of the horizontal impact load of the aircraft wheel, $P(t)$.

\subsection{Drop test apparatus and measurement of the transient rotational speed of an aircraft wheel}

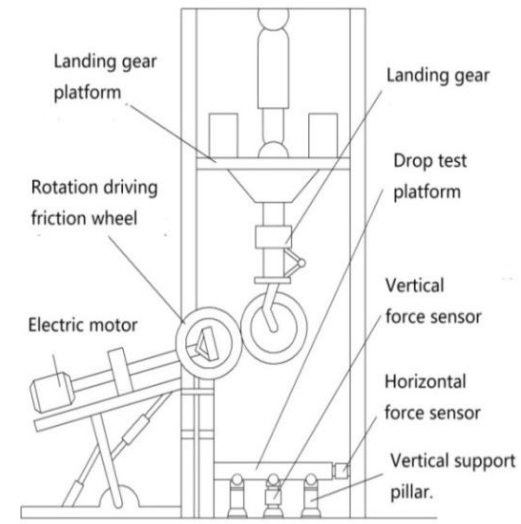

Figure 2 Schematic of the drop apparatus

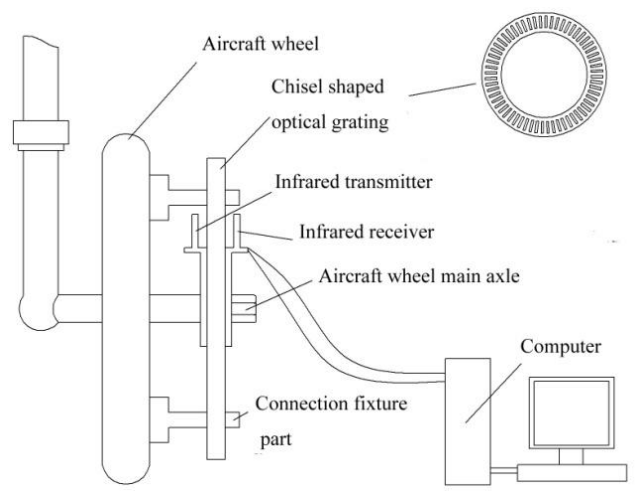

Figure 3 Rotational speed measurement system test for the landing gear wheel

The basic structure of the traditional column-style landing gear drop test apparatus is shown in Figure 2. It is mainly composed of three components, including a landing gear platform that can slide freely up and down, an apparatus for driving the rotation of the aircraft wheel, and a drop test forcemeasuring platform. In the drop test process, the sudden change in the rotational speed of the aircraft wheel upon contact with the ground is enormous. Within a few tenths of a second, the rotational speed of the aircraft wheel can be reduced from several thousand revolutions per minute to tens of revolutions per minute. The high-speed impact experienced by the landing gear at the instant of contact with the ground causes the landing gear to generate a wide range of elastic impact displacements in three-dimensional space; these displacements range from tens of millimeters to hundreds of millimeters and are accompanied by intense vibration, making the testing environment adverse. For this reason, a non-contact apparatus for measuring the rotational speed of the aircraft wheel, composed of a chisel shaped optical grating, an infrared transmitter, an infrared receiver, a 
connection fixture part, and a computer, was designed and installed on the landing gear wheel, as shown in Figure 3.

The process of measuring the rotational speed of the aircraft wheel is as follows: when the landing gear wheel drops at a rotational speed of several thousand revolutions per minute, a relative rotational displacement is generated between the infrared transmitter and receiver installed on the landing gear main axle and the grating disc installed on the aircraft wheel. Because the grating is an equidistant light transmission grating, the infrared receiver can receive the optical pulse signal associated with the changes in aircraft wheel rotational speed and then convert the optical pulse into an electrical pulse. Concurrently, the computer records the time interval between each adjacent pulse received by the infrared receiver. Because the chisel shaped optical grating contains equally spaced planes, the angle between the adjacent holes in the optical grating is a fixed constant; therefore, the change in the rotational speed of the aircraft wheel in the impact process can be computed from the recorded relationship between the rotation angle and the pulse interval time[6].

The horizontal impact load of the aircraft wheel can be determined by substituting the measured rotational speed of the aircraft wheel and related parameters into Formula (3).

\section{Determining the horizontal impact load and experimental verification}

\subsection{Test apparatus}

To determine the horizontal impact load of the aircraft wheel in the landing gear drop test and to verify the accuracy of the method used to determine the horizontal impact load from the measured rotational speed of the aircraft wheel, a drop test simulation apparatus was designed and built, as shown in Figure 4.

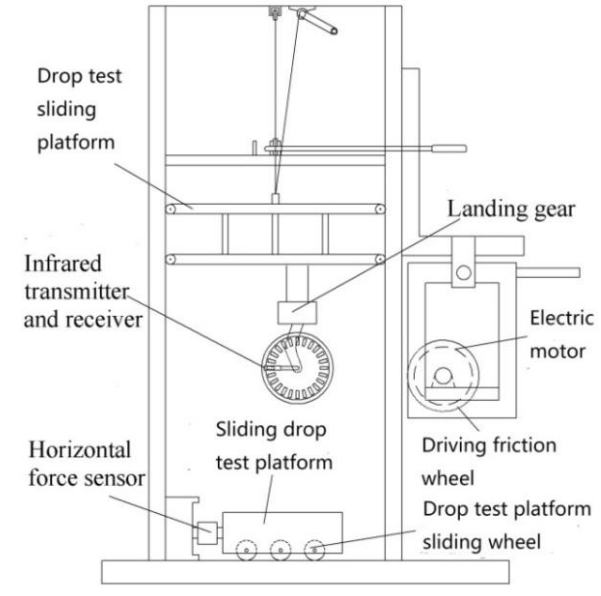

Figure 4 Drop test simulation and verification apparatus

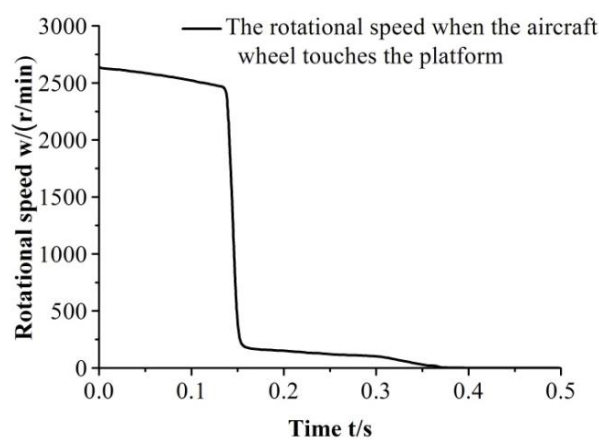

Figure 5 Curve of the rotational speed when the aircraft wheel touches the platform

A sliding drop test platform composed of a rolling bearing was set up at the base platform of the test simulation apparatus. This allowed the platform to move freely in the horizontal direction. A horizontal force sensor was installed at one end of the sliding drop test platform. One side of the sensor was fixed and connected to the support column of the test apparatus by a bolt, and there is no lateral constrained structure for the sliding drop test platform in the lower part of the test device. The horizontal friction generated between the aircraft wheel and the platform when the aircraft wheel is dropped and makes contact with the platform while under high-speed rotation is then transmitted to the horizontal force sensor through the sliding platform. Because this test apparatus only uses one horizontal force sensor, it avoids the mutual restraint and coupling effect in installation and measurement between the sensors that measure multiple dynamic strength parameters in large drop tests. The data measured by the horizontal force sensor represent the horizontal impact load of the 
rotating aircraft wheel upon contact with the platform. The apparatus is equipped with a rotational speed measurement system focused on the aircraft wheel; this system can simultaneously record the change in rotational speed when the aircraft wheel is allowed to drop and contact the platform.

\subsection{Measurement results on the transient rotational speed of the aircraft wheel, $\omega$}

To collect the measurements, first, the landing gear wheel was driven by an electric motor to rotate at a high speed; then, the release apparatus was initiated to drop the landing gear into a vertical free-fall while the computer collected the electric pulse signals generated by the rotation of the chisel-shaped optical grating. The computer then calculated and completed the measurement while recording the transient rotational speed of the aircraft wheel.

Figure 5 is a curve of the measured change in the transient rotational speed of the aircraft wheel during the test. Because the optical grating was arranged tightly, the number of optical gratings could reach 180 or higher per revolution; therefore, the curve of the collected change in the rotational speed of the aircraft wheel when the aircraft wheel touched the platform was smooth. According to Figure 8, the rotational speed of the aircraft wheel suffers a certain amount of attenuation due to the effect of the friction torque of the axle from the drop until prior to touching the platform. Within approximately 0.15 seconds after release, the rotational speed of the aircraft wheel decreased from $2,630 \mathrm{r} / \mathrm{min}$ at the time of separation from the rotation driver to $2,460 \mathrm{r} / \mathrm{min}$ at the time of contact with the platform; in total, the rotational speed decreased by approximately $6.5 \%$. At the instant of contact with the platform, the intense friction between the aircraft wheel and the platform caused a sudden decrease in the rotational speed of the aircraft wheel; hence, within an extremely short time, i.e., less than 0.03 seconds, the rotational speed rapidly decreased from $2,460 \mathrm{r} / \mathrm{min}$ to $165 \mathrm{r} / \mathrm{min}$, representing an approximate decrease of $94 \%$. After this point, the rotation of the aircraft wheel stopped at 0.4 second.

\subsection{Measurement results of the rotational speed, $\omega_{0}$, of the aircraft wheel in a state of free rotation attenuation}

To obtain the rotational speed, $\omega_{0}$, during free attenuation of the aircraft wheel under the effect of the friction torque of the axle, $M$, in Formula (3), the landing gear wheel was driven to spin at the release rotational speed; then, the rotation-driving friction wheel was separated from the aircraft wheel, although the aircraft wheel was not allowed to drop, so that the aircraft wheel freely attenuated only under the effect of the friction torque of the axle. Meanwhile, the rotational speed measuring system was triggered to collect and record the rotational speed of the aircraft wheel in the free attenuation process.

The moment of inertia, $I$, of the aircraft wheel needed in Formula (3) can be determined using a torsion pendulum method[7].

\subsection{Determining the horizontal impact load from the measurement results of the measured rotational speed of the aircraft wheel, $\omega$ and $\omega_{0}$}

The measurement results of the transient rotational speed of the aircraft wheel, $\omega$, and the rotational speed of the aircraft wheel in a state of free attenuation, $\omega_{0}$, the moment of inertia of the aircraft wheel, and the value of the aircraft wheel radius were substituted into Formula (3). Through computation, the curve identifying the change in the horizontal impact load over time when the aircraft wheel touched the platform was obtained as shown in Figure 6.The figure indicates that within 0.012 second after the aircraft wheel made contact with the platform, the horizontal impact load rapidly increased from zero, reaching a maximum value at 0.146 second. Subsequently, the horizontal impact load continued to decrease and tended toward zero. 


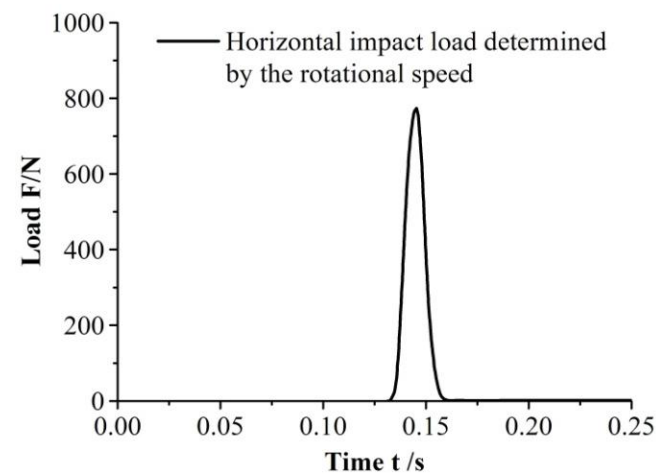

Figure 6 Curve of horizontal impact load determined by the measured rotational speed of the aircraft wheel

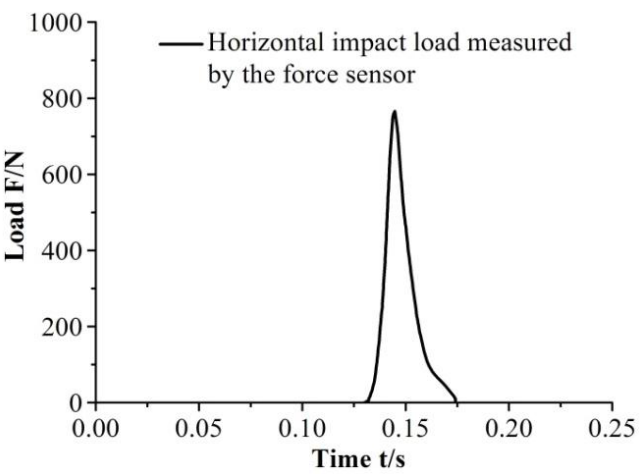

Figure 7 Curve of the horizontal impact load as measured by the force sensor

\subsection{Results of horizontal impact load measured by force sensor}

During the experiment, the landing gear wheel was first driven to rotate at a high speed by an electric motor, and then, the aircraft wheel was driven to rotate by friction to approximately $2,700 \mathrm{r} / \mathrm{min}$. The release apparatus was started to induce a vertical free-fall of the aircraft wheel, while the computer completed the measurement and recording of the transient rotational speed of the aircraft wheel and the horizontal impact load.

The measurement results of the horizontal force sensor are shown in Figure 7. The curve in the figure reflects the changes that occurred in the horizontal impact load when the aircraft wheel contacted the platform. At the instant that the aircraft wheel made contact with the sliding platform, due to the rapid reduction in rotational speed from the large frictional force, the horizontal impact load increased significantly, reaching a maximum value of $744.36 \mathrm{~N}$ at approximately 0.012 second after the time of contact between the aircraft wheel and the platform. Subsequently, the horizontal impact load reduced to $110.23 \mathrm{~N}$ within 0.016 second, representing a decrease of approximately $85 \%$. After another 0.017 second, the horizontal impact load neared zero.

\subsection{Comparison of the horizontal impact load results determined by the rotational speed and the direct measurement results obtained with the force sensor}

To compare the horizontal impact load determined by the measured rotational speed of the aircraft wheel with the horizontal impact load measured by the horizontal force sensor, Figure 8 shows the curves of the horizontal impact load obtained by both methods in the same experiment. According to the figure, both methods obtained the same trends in the changes of the horizontal impact load. The peak value of the horizontal impact load determined by the measured rotational speed of the aircraft wheel was $772.42 \mathrm{~N}$, and the peak value of the horizontal impact load measured by the horizontal force sensor was $744.36 \mathrm{~N}$; the relative error between the two was $3.77 \%$, which indicates that this approach is able to meet the requirements of actual engineering application. The results of the above comparison indicate that the method in which the horizontal impact load is determined based on the measured transient rotational speed of an aircraft wheel is effective and feasible.

According to the friction deceleration principle, the different contact materials and different surface qualities between the aircraft wheel and the ground generate different sizes and courses in the horizontal impact load of the aircraft wheel. Moreover, the process of change in different horizontal impact loads corresponds to the process of change in the rotational speed of the aircraft wheel. By establishing the kinetic relationship between the force of the horizontal impact and the transient rotational speed of the aircraft wheel, this paper measured the dynamic change in the rotational speed of the aircraft wheel at the instant of aircraft contact with the ground and, from this, was able to determine the size of the horizontal impact load of the aircraft wheel. 


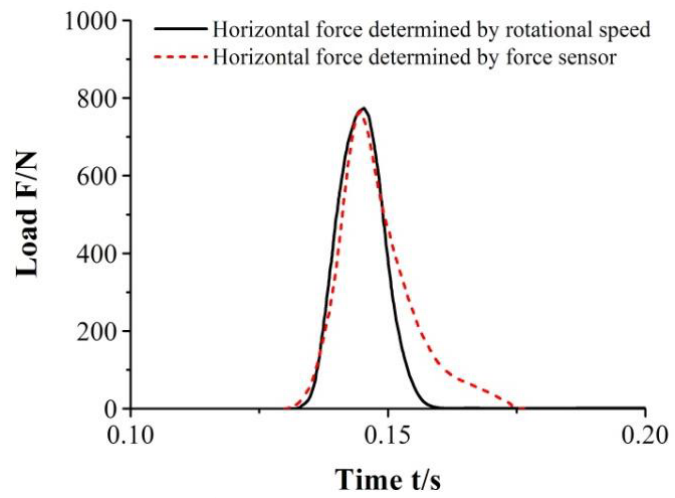

Figure 8 Comparison of horizontal impact load values obtained by two different measurement methods

\section{Conclusion}

By establishing the kinetic relationship between the transient rotational speed of the aircraft wheel and the force of the horizontal impact, This paper proposes a method that the horizontal impact load of the aircraft wheel can be determined based on the measured dynamic change in the rotational speed of the aircraft wheel at the instant at which the aircraft contacts the ground. The maximum values obtained by the method of using the measured rotational speed of the aircraft wheel to determine the horizontal impact load proposed in this paper match well with the measured horizontal impact load recorded by using horizontal force sensors. This demonstrates the accuracy and usability of this method in which the measured transient rotational speed of the aircraft wheel determines the horizontal impact load.

\section{Acknowledgements}

This work was supported by the National Natural Science Foundation of China under Grant No. 11427802 .

\section{References}

1. Besch, Hans Martin. Large aircraft landing gears-a brief overview. 6th International Munich Chassis Symposium 2015. Springer Fachmedien Wiesbaden, 2015:685-715.

2. Wei,W.U."Study On Load Measurement And Calibration Test For Airplane Landing Gear." Journal of Mechanical Strength (2003).

3. Zhang, Daqian. "The present situation and development of chinese drop test measurement and test system." Journal of Shenyang Institute of Aeronautcal Engineering (2002).

4. Xue Caijun, et al. "Optimization of the cushion properties of amphibious aircraft landing gear by simulation and drop test." Journal of Vibration \& Control 19.19(2013):1753-1760.

5. SHI Hai-wen, et al."Parameters Study at Touchdown Instant of the Nose Undercarriage of Fighter Airplane." Acta Aeronautica et Astronautica Sinica 24.6(2003):517-520(In Chinese).

6. WU DaFang, LI YongTing, WANG YongHai, SONG Hao. Measurement method of the transient rotational speed of landing gear on drop test. Journal of Experimental Mechanics, 2008

7. W. Bin, M. GuiXian, P. MinYan. A Measurement Method of Moment of Inertia for the Artificial Satellite. Journal of Astronautic Metrology and Measurement, 2008, 28: 21-23 (In Chinese). 